\title{
Measuring acute stress response through physiological signals: towards a quantitative assessment of stress.
}

\author{
Adriana Arza ${ }^{1,2,3^{*}}$, Jorge Mario Garzón-Rey ${ }^{1,2}$, Jesús Lázaro ${ }^{1,4,5}$, Eduardo Gil ${ }^{1,4}$, Raul Lopez-Anton ${ }^{5}$, \\ Conchita de la Camara ${ }^{7}$, Pablo Laguna ${ }^{1,4}$, Raquel Bailon ${ }^{1,4}$ and Jordi Aguiló ${ }^{1,2,8 *}$ \\ ${ }^{1}$ CIBER de Bioingeniería, Biomateriales y Nanomedicina (CIBER-BBN), Zaragoza. \\ ${ }^{2}$ Microelectronics and Electronic Systems Department. Autonomous University of Barcelona, Bellaterra, Spain \\ ${ }^{3}$ Embedded System Laboratory, Swiss Federal Institute of Technology Lausanne (EPFL), Lausanne 1015, Switzerland \\ ${ }^{4}$ BSICoS Group, Aragon Institute of Engineering Research (I3A), IIS Aragón, University of Zaragoza, Spain. \\ 5 Department of Biomedical Engineering, University of Connecticut, Storrs CT, USA \\ ${ }^{6}$ Psychology and Sociology Department of University of Zaragoza, Spain. \\ ${ }^{7}$ Psychiatric Service of Zaragoza Clinical Hospital, Spain. \\ ${ }^{8}$ Microeletronics National Centre. IMB-CNM, CSIC Barcelona Spain \\ *Corresponding Authors: \\ E-mail: adriana.arza@epfl.ch (AA) \\ jordi.aguilo@uab.cat (JA)
}

\begin{abstract}
-
Social and medical problems associated with stress are increasing globally and seriously affect mental health and wellbeing. However, an effective stress-level monitoring method is still not available. This paper presents a quantitative method for monitoring acute stress levels in healthy young people using biomarkers from physiological signals that can be unobtrusively monitored. Two states were induced to 40 volunteers, a basal state generated with a relaxation task and an acute stress state generated by applying a standard stress test that includes five different tasks. Standard psychological questionnaires and biochemical-markers were utilized as ground truth of stress levels. A multivariable approach to comprehensively measure the physiological stress response is proposed using stress biomarkers derived from skin temperature, heart rate and pulse wave signals. Acute physiological stress levels (total-range 0-100au) were continuously estimated every one-minute showing medians of 29.06 au in the relaxation tasks, while rising from 34.58 to 47.55 au in the stress tasks. Moreover, using the proposed method, five statistically different stress levels induced by the performed tasks were also measured. Results obtained show that, in these experimental conditions, stress can be monitored from unobtrusive biomarkers. Thus, a more general stress monitoring method could be derived based on this approach.
\end{abstract}

Keywords: Stress measurement; Stress biomarker; Multi-modal analysis; Multivariable biomarker; Acute stress; TSST; Unobtrusive physiological signals. 


\section{Introduction}

The global incidence of anxiety, depression, multiple sclerosis, pathological stress and different stress-related diseases has significantly increased in recent years; this is probably due to dramatic changes in the daily lives of citizens. It has been shown that stress generally has negative consequences in terms of mental health and wellbeing [1-3] and that chronic stress especially increases the risks of certain pathologies, such as cardiovascular diseases, strokes, diabetes and others $[4,5]$ and can even cause sudden death [6]. According to the World Health Organisation [7], social and medical problems associated with stress are clearly rising and seriously affecting mental health and well-being not only in adults but also in young people and children. Therefore, stress has become one of the significant focal points of research interest [8]. Reliable markers that allow to measure the body's stress response could be utilized to monitor stress conditions in daily life. This would, in turn, enable an accurate monitoring of patients, prevent illnesses and detect pathological conditions in their early stages. However, to the best of our knowledge, no reliable tool yet exists for measuring levels of stress response.

Stress has been defined by Selye in 1950 as a state of biological activation triggered by the person interacting with external agents that force her or his capacity to adapt [9]. Stress is also defined as a disturbance of homeostatic balance of the individual [10] that provokes a stress response, which is the body's (or individual's) attempt to cope with the stressor. Stress can be classified as either acute or chronic [3,11]. Acute stress is the immediate body's response to a stressor. Chronic stress, on the other hand, is the state caused by a continuous stressor stimulus over a period of months (e.g. family caregivers of terminal patients) or the state reached when the body cannot to deal with the stressor and does not achieve a homeostatic balance state (e.g. traumatic stress of war veterans).

The response to acute stress is designed to provide the organism with the requisite alertness, energy, physiological regulation and immunological activation to counterbalance the effects of the stressor in order to reach a new adaptive state and survive [11]. Consequentially, the activation of stress response triggers changes in the autonomous nervous system (sympathetic and parasympathetic balances) and a hormonal response, as well as behavioural changes and a decrease in cognitive skill $[3,11]$. Thus, a diversity of body changes is triggered, affecting different possible stress markers, which have been used to assess stress so far.

Psychiatry, psychology and the medical community in general have established and are using, psychometric questionnaires as the appropriate tools to assess psychological stress [12], measuring mostly behavioural and cognitive skill changes. Among others, one of the most frequently used questionnaires are the subject's self-reporting assessments (e.g. the Visual Analogue Scale for Stress, VASS). Following a different approach, researchers from biomedical fields are more enthusiastic about using biochemical markers from the hormonal response, such as cortisol, prolactin, $\alpha$-amylase [13], interleukin-6, copeptin [14] and others [15, 16]. However, even though biochemical samples and psychometric questionnaires constitute well-known stress makers, they cannot be implemented in a continuous stress monitoring method. Nevertheless, they are suitable for specific applications and research studies. The limitations of these biochemical markers include invasiveness and the impossibility of being continuously measured. Additionally, it must be noted that their dynamic response should be considered in order to perform a proper measurement [17]. The relationship between the body's activation of biochemical markers and the 
intensity of the stress perceived is both complex and understudied, which also makes the use of biochemical markers disadvantageous. The major drawbacks of the psychometric questionnaires are that they are not designed for frequent applications and they are subjective, depending on the self-perception of the individual about her or his condition.

On the other hand, diverse studies exist that assess stress using mainly markers extracted from biosignals and thus measure the physiological reactions of the stress response on the body (see $[12,18-20]$ ). The most widely used biosignals are electrocardiography (ECG) [21, 22] and skin conductance (SC) [21, 23]. Some features extracted from these biosignals, such as the heart rate (HR) and mainly its variations (HRV), are driven by the autonomic nervous system (ANS) and, more precisely, by its sympathetic and parasympathetic components; thus, they constitute good stress markers. Other biosignals are also used such as electroencephalogram [24], skin temperature [25], pulse photoplethysmography (PPG), respiration [21, 22], pupil diameter, electromyography and blood pressure.

Most of these studies employed similar approaches. Firstly, while some physiological signals are measured, a stress state is induced under a real situation $[21,23,26-28]$ or in a laboratory $[22,24,29,30]$, by, for example, a mental arithmetic [22, 23], the Stroop Test, film fragments, or the Trier Social Stress Test [25]. Subsequently, a variety of features are used with either pattern recognition or machine learning techniques that allow the automatic classification of stress states. The applied techniques are also diverse, such as support vector machines [22], fuzzy logic, Bayesian networks, decision trees [23], artificial neural networks [31] and computer aided diagnostic tools [32]. Notwithstanding, most of these studies only allow stress detection (stress/ no stress) [23] and few of them identify three levels of stress $[22,29,30]$, i.e. high, medium and low, normally related to the intensity of the stressor. Additionally, the methods that have been employed as the ground truth of the induced stress intensity reference are diverse. Several studies employ standard psychometric questionnaires [22, 24] or subject's self-report assessments as a reference, regardless its previous mentioned limitations. Other studies employ as a reference the status of the stressor stimulus, i.e. active or not [33], while others use the amount of work load and cognitive demand that is being applied as the stressor agent [21].

Despite the efforts made, a scientifically supported, objective, reliable, repeatable and easily usable measurement method remains unavailable. Indeed, up to now, no agreement among professionals or within the research community exists, neither on a unique stress assessment method nor in a standard stress measurement method.

We propose to use only physiological biomarkers that can be unobtrusively monitored, aiming to obtain a reliable description of the stress response that can be easily monitored in daily life. Therefore, the main objectives of this study are the following: (1) to identify a set of features derived from physiological signals that comprehensively describe the physiological stress response according to established stress markers; and, (2) to then use them for a reliable quantitative assessment of stress level as a first attempt towards stress monitoring. Although the ultimate goal is to measure acute stress or chronic stress in daily life, a potential stress measurement method should first be validated under controlled stress conditions. Thus, this work contributes to the extant literature by:

- Designing an experiment to quantify participants' levels of acute psychological induced stress

- Selecting a set of stress biomarkers from physiological signals that describe the stress response level in a similar range as do the standard psychological questionnaires and biochemical stress markers 
- Proposing a multimodal approach for stress-levels measurement based on a comprehensive assessment of the physiological response of the individual to a stressor agent

- Proving that, in these experimental conditions, stress response levels can be unobtrusively and continuously measured from a set of physiological signals, as a viable alternative to the currently used stress assessment methods.

\section{Methods}

The research presented in this paper involves experimental measurements of physiological signals, biochemical variables and psychometric questionnaires in healthy young students from the Autonomous University of Barcelona (UAB), who were under an acute psychological standard stressor.

Varieties of stressor stimuli have been employed in stress research. The Trier Social Stress Test (TSST) has been chosen in this study to induce acute psychological stress because TSST is one of the most widely used and documented protocols in stress research [34-37] ${ }^{\text {and }}$ because it provides a robust and reliable acute stress inducement [38] compared with other stressors.

Two main stress states were induced to the participants, a basal state (BS) and an acute stress state (SS). Participant's stress response was assessed using a set of stress reference variables (well-established stress markers). Suitable features derived from the physiological signals were selected and compared with a whole set of stress reference variables. Afterwards, these features were used to make an estimation of stress level.

\subsection{A Multimodal Approach to Stress Measuring}

The methodology and analysis of the results presented are focused on objectively quantifying the physiological components of the stress response, which can be understood as the degree of disturbance of homeostasis [11], i.e., how close or far an individual is from the state of homeostatic balance. The response to stress triggers biological changes that prepares the body to deal with a stressor agent, which provoke the homeostatic imbalance that will continue until the achievement of a new state that is adapted to the new situation.

Consequently, the stress level response is determined as a function of the particular reactions that the homeostatic imbalance provokes in the individual. Such stress reactions are dependent not only on the exposure time to stressors (acute or chronic), but also on certain characteristics of the stressor as they are perceived by the individual, such as its intensity, her or his own ability to control it and its predictability [39].

The physiological reactions of the homeostatic imbalance triggered by the stress response can be broadly summarized as follows [35]:

1) Neurohypophysis activation: vasopressin secretion leading to increased blood volume and pressure and increased peripheral blood vessels resistance

2) Hypothalamic-pituitary-adrenal axis (HPA axis) activation: cortisol secretion leading to increased catabolism, anabolism inhibition as well as immune system depression

3) Sympathoadrenal system activation: sympathetic nervous system sensitization 
4) Nervous system activation: sympathetic nervous system activation and parasympathetic withdrawal, causing increased heart rate and respiratory frequency, bronchial and pupil dilation, sweating skin, peristalsis inhibition and hyperglycaemia and other symptoms.

Thus, a single stress marker cannot globally assess the stress response of an individual, as it is also concluded in [20]. In order to be able to compare and assess the stress level response, a method that considers the different stress response reactions as a whole is requisite. Therefore, a multivariable approach is proposed in this study to assess the individual stress response to a stressor.

\subsection{Study Design}

The study is a quasi-experimental pre-post study without a control group to elucidate the effect of a stressor on psychological, biochemical and physiological stress markers in a unique group of healthy students. The experiment design included a Relax Session, as a control condition and a Stress Session, performed on different dates, so that participants could be compared to themselves in a basal state and a stress state.

A preparatory relaxation period of $10 \mathrm{~min}$ was conducted at the start of each session to achieve a fairly similar baseline state among participants for both sessions. The state reached after the Relax Session was considered as the basal state while the state reached at the end of the Stress Session was considered as the stress state. During both sessions, physiological signals were continuously recorded while standard psychological questionnaires were applied and biochemical markers collected at the end of each session, as shown Fig. 1. The experimental design shares its measurement framework with other studies within the ES3 Project [8], which aims to cover all complexities of stress.

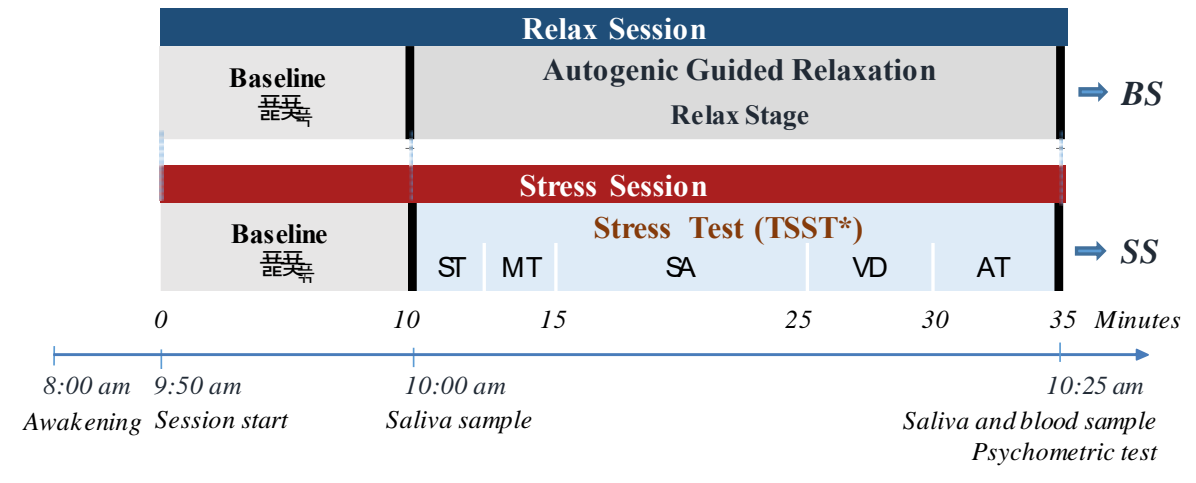

Fig. 1 Details of study protocol. The first saliva sample was taken after the baseline stages (BLR, BLs) and the second saliva sample at the end of the Relax and the Stress Sessions. A blood sample was taken and psychometric tests were applied at the end of each session. Physiological signals were measured during both, the Relax Session and during the Stress Session stages: story telling (ST), memory test (MT), stress anticipation (SA), video display (VD) and arithmetic task (AT). Stress states reached at the end of the Relax and the Stress Sessions were basal state (BS) and acute stress state (SS), respectively.

\subsubsection{Participants}

Participants were recruited using an advertisement on the UAB website, which was linked to the webpage of the ES3-Project [40]. All interested candidates completed an online form beforehand, to ensure that they met the selection criteria for the study. After individually checking to determine whether they fulfilled the inclusion 
requirements, participants were included in the study consecutively according to when they were enrolled in the study. Appointments were made at random.

The inclusion criteria were to be between 18 and 30 years old and a non-regular consumer of psychotropic substances, alcohol, or tobacco. The exclusion criteria were a body mass index higher than 30, any chronic disease or psychopathology and a stress level higher than $70 \%$ on a visual analogue scale.

The UAB Ethics Committee approved the study protocol. Participants gave their written informed consent. They were instructed to avoid the use of any psychotropic substance, alcohol or tobacco and to avoid doing any physical exercise for $24 \mathrm{~h}$ prior to each session, wake up $2 \mathrm{~h}$ before the start of the sessions and consume a light breakfast without coffee or tea.

\subsection{Experimental Protocol}

The Relax and the Stress Sessions were performed on different dates, but at the same hour for each participant. The Relax Session comprised two stages, the baseline stage $\left(B L_{R}\right)$ and the relax stage $(R S)$, while the Stress Session had a baseline stage $\left(\mathrm{BL}_{\mathrm{S}}\right)$ and five different stages, inducing acute psychological stress through a standard stressor.

The relaxation was induced by autogenic relaxation guided by an audio recording conforming to Schultz's method [41] at stages $\mathrm{BL}_{\mathrm{R}}$ and $\mathrm{RS}$ in the Relax Session and $\mathrm{BL}_{\mathrm{S}}$ in the Stress Session.

Acute psychological stress was generated with a modification of TSST as follows. Instead of the speech, the participants performed a memory test while being video-recorded. Then, the video was shown to her or him in the company of an audience, after a prior stress anticipation period [42]. This modification was introduced to compare the results from the Memory Test with the study performed in [42] as part of the ES3 Project [8].

The stress test included five different stages over $25 \mathrm{~min}$. Each stage had different demanding conditions, inducing different stress states in participants:

1) Story telling stage (ST): three different short stories are told to the subjects and they are asked to remember as many details as possible.

2) Memory test stage (MT): The subjects are requested to repeat each story aloud, including as many details as they can in a 30 -second period per story. During this stage, the subjects are notified that they will be videorecorded to assess their performance.

3) Stress anticipation stage (SA): The participants are asked to go to an adjacent, empty room under the pretext of they should wait until the group of psychologists assesses their video.

4) Video display stage (VD): The participants return to the previous room, where an unexpected audience of at least three people is waiting for them. The video showing each participant's storytelling is played in front of the audience. Each video is interwoven with another video, in which an actor of the same gender is telling most of the details of each story, to increase the subject's arousal.

5) Arithmetic task stage (AT): The subjects are asked to count backwards from 1022 in increments of 13, in less than $5 \mathrm{~min}$. If they make a mistake, they should start again from 1022. 
At the end, the subject is required to complete the psychometric questionnaire. Participants were seated the entire time, except at the beginning and the end of the atress anticipation stage, when they walked into the adjacent room.

\subsection{Physiological Signals: Acquisition and Processing}

Electrocardiogram, pulse photoplethysmography, respiration, skin conductance and face and finger temperature signals were selected due to their relation with stress response and because they can be continuously and unobtrusively monitored. The main aspects concerning signal selection, processing and feature extraction methods used in this research are listed below. Among all those physiological signals that show changes related to stress [8], the signals that can be unobtrusively monitored and that comprehensively describe the stress response were selected. They are presented in Table 1.

Table 1. Selection of physiological signals

\begin{tabular}{lll}
\hline \hline Physiological measures & Measurable physiological manifestation to stress response & Sensor body position \\
\hline Peripheral skin temperature & $\begin{array}{l}\text { Neurohypophysis activation and sympathetic nervous system } \\
\text { activation }\end{array}$ & Finger \\
\hline Core skin temperature & $\begin{array}{l}\text { Neurohypophysis activation and sympathetic nervous system } \\
\text { activation }\end{array}$ & Cheek \\
\hline Skin conductance & Sympathetic nervous system activation & Hand \\
\hline Electrocardiogram & $\begin{array}{l}\text { HPA axis activation, sympathetic nervous system activation and } \\
\text { parasympathetic counterbalance }\end{array}$ & 3 Orthogonal leads in \\
& the thorax \\
\hline Respiration rate & $\begin{array}{l}\text { Sympathetic nervous system activation and parasympathetic } \\
\text { counterbalance }\end{array}$ & Thorax \\
\hline Photoplethysmography & Neurohypophysis activation, HPA axis activation, sympathetic & Index finger \\
\hline \hline
\end{tabular}

The ECG signal was sampled at $1 \mathrm{kHz}$, while the other signals were sampled at $250 \mathrm{~Hz}$ using the Medicom system, ABP-10 module (Medicom MTD Ltd, Russia). Most recordings of skin conductance had to be discarded in the reported experiment due to a sensor malfunction. Thus, skin conductance values were not considered in any further analyses. Respiration signal was also excluded from this analysis, as it is clearly artefacted during the MT and AT stages when the participant had to talk.

\subsubsection{Skin Temperature}

Variations in face and finger temperature are associated with stress response due to the activation of the vasoregulatory system (i.e. a peripheral vasoconstriction together with a vasodilatation in the core and face) [25]. Both face $\left(\mathrm{T}_{\mathrm{fa}}\right)$ and finger $\left(\mathrm{T}_{\mathrm{fi}}\right)$ temperature signals were filtered using a one-dimensional median filter (50 orders) and then values out of the $20-40^{\circ} \mathrm{C}$ range were excluded. Subsequently, the following features were computed:

- Temperature gradient $(\Delta \mathrm{T})$ : temperature variation in sliding windows of 1 min time

- Temperature power (T_Ptotal): defined as the average power of temperature measured via a rectangle approximation of the integral of the power spectral density in sliding windows of one-minute time 
- Temperature ratio $\left(\mathrm{T}_{\mathrm{ratio}}\right)$ : ratio between mean values of finger $\left(\mathrm{T}_{\mathrm{fa}}\right)$ and face $\left(\mathrm{T}_{\mathrm{fi}}\right)$ temperatures in sliding windows of 1 min time

\subsubsection{Electrocardiogram}

The ECG signal was analysed as in [43] to obtain a time series of R to R-peak (RR series). The following features were computed, which are explained in detail in [44]:

- Time features: From the fixed RR series, mean heart rate $\left(H_{\mathrm{m}}{ }_{\text {men }}\right)$, standard deviation of all $\mathrm{RR}$ Intervals $\left(R_{\text {SDNN }}\right)$ and root mean square of successive differences of $R R\left(R_{r M S S D}\right)$ were computed;

- Frequency features: Low frequency (LF) power (PLF) and high frequency (HF) power (PHF), the LF/HF ratio and power in the very low frequency (PVLF) band.

\subsubsection{Pulse Photoplethysmogram}

A filter was applied to the PPG signal, as well as an artefact detector as described in [45]. Fiducial points in pulse waves were automatically determined from each pulse beat by an implemented algorithm based on [46]. The detected points for the $i^{\text {th }}$ pulse in PPG signal are seen in Fig. 2.

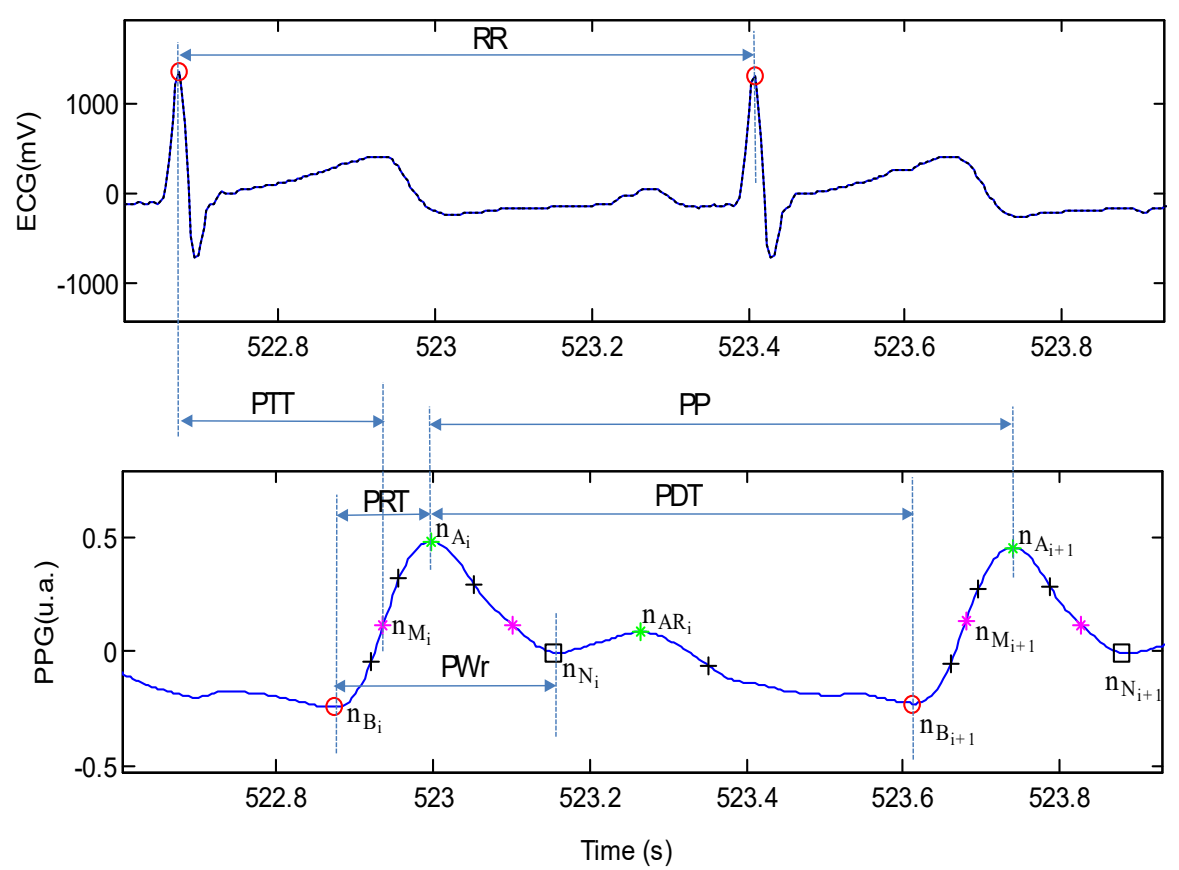

Fig. 2 Detection points in ECG and PPG signals. Each absolute maximum of the PPG signal between two successive QRS complexes detected in the ECG signal is considered an apex point $n_{A i}$ of a PPG pulse. $n_{B i}$ : the basal point is the absolute minimum of the PPG signal between two successive QRS complexes in the ECG signal. $n_{\text {Mi }}$ : the middle point is the instant in time when the PPG pulse reaches half of its apex-to-basal amplitude. $n_{A R i}$ : the apex point of the reflected wave is the maximum reflected pulse wave. $n_{N i}$ : the dicrotic notch point is the inflection point between the pulse wave and the reflected wave.

Pulse period $\left(\mathrm{PP}_{\mathrm{i}}\right)$ is the time interval between two consecutives $n_{A i}$ and the Pulse rate $\left(\mathrm{PR}_{\mathrm{i}}\right)$ is the inverse of the $P P_{i}$ series. A subsequent analysis to obtain pulse period features was equal to the aforementioned HR time features from 
the ECG signal ( $\left.\mathrm{PR}_{\text {mean }}, \mathrm{PP}_{\mathrm{rMSSD}}, \mathrm{PP}_{\mathrm{SDNN}}\right)$. Furthermore, additional time-based parameters were calculated (see Fig. 2) and their median values and standard deviations in sliding windows of 1 min time were assessed:

- Pulse transit time (PTT). The PTT is inversely proportional to pulse wave velocity, which is associated with arterial stiffness and cardiovascular output. Therefore a decrease in PTT is related to an increase in blood vessel resistance and cardiovascular output, as well as being inversely related to blood pressure [47].

- Pulse wave rising time (PRT). The PRT is proportional to the pulse amplitude and is related to arterial stiffness, blood volume and systolic blood pressure peak [48].

- Pulse wave decreasing time (PDT). The PDT is an index of diastolic time, which is also highly related to PR.

- Pulse width until reflected wave (PWr): The PWr is a time index of the reflected pulse wave arrival and is associated with the PRT, the peripheral central vascular resistance, the cardiovascular output and the pulse wave velocity [49].

\subsection{Acquisition and Analysis of Stress Reference Variables}

The stress reference variables were selected from established psychometric questionnaires and biochemical stress markers taking into account a bibliographic review conducted and the criteria of medical doctors and psychologist involved in the ES3 Project, as described in [8]. These variables are used to assess the stress state reached at the end of each session, the basal state (BS) and stress state (SS), respectively.

\subsubsection{Psychometric Variables}

The psychometric questionnaires applied were the Perceived Stress Scale (PSS), the Visual Analogue Scale (VASS) and the State-Trait Anxiety Inventory test (STAI). The PSS measures the degree of overall stress of the individual or the extent to which life situations are appraised as stressful [50]. The VASS records the stress level that is self-perceived by the participant. The STAI evaluates anxiety from two different points of view: (1) as a measure of the subject's state (STAI-s) at a given time and (2) as the trait (STAI-t) or stable tendency of the individual to respond by increasing her or his level of anxiety in stressful situations [51]. These questionnaires were selfadministered at the end of each session.

\subsubsection{Biochemical Variables.}

Biochemical markers collected were copeptin and prolactin obtained from blood samples and cortisol and $\alpha$ amylase obtained from saliva samples. In accordance with the circadian rhythm of cortisol and $\alpha$-amylase [52], the sessions were scheduled in the morning (09:45-10:45h), $2 \mathrm{~h}$ after waking up. Two saliva samples were taken in each session: one before the stimulus (relaxation or stress) and the other at the end of the session [53] (i.e. 25 min inbetween) to obtain cortisol ( $\Delta$ cortisol) and $\alpha$-amylase ( $\Delta \alpha$-amylase) variation per session, see Fig. 1.

Cortisol and $\alpha$-amylase were measured in the endocrinology and radioimmune analysis service of the UAB Neurosciences Institute. An immunoassay was performed to determine cortisol concentration and an enzyme assay analysed the $\alpha$-amylase enzyme kinetics (Salimetrics, State College, PA, U.S.A.). The analysis of prolactin and copeptin was quantified at the Centre of Biomedical Diagnostic at the Clinic Hospital of Barcelona. All of the 
samples were processed in the same test to avoid any inter-test variability, achieving intra-test variation coefficients lower than $5 \%$ in both cases.

\subsection{Stress Reference Level}

The stress reference variables were used as a reference to compute a stress reference scale applying the multivariable approach presented in Section 2.1. The stress references variables were re-scaled to a 0-100 range of arbitrary units. The stress reference level of participant $\mathrm{p}$, in state $\mathrm{x}$ (BS or SS), $S_{\text {ref }}(p, x)$, is described as a linear combination of $n$ stress reference variables [54], as expressed by the following equation:

$$
S_{r e f}(p, x)=\sum_{j=1}^{n} c_{j} \cdot S R V_{j}^{\prime}(p, x) \quad ; x \in\{B S, S S\}
$$

where $S R V_{j}^{\prime}(p, x)$ is the re-scaled value of the $j$ th reference variable for the participant $\mathrm{p}$ at state $\mathrm{x}$; the coefficient $c_{j}$ represents the contribution of each $S R V_{j}^{\prime}$ to the state's differentiation. This coefficient was assigned by using a ponderation of the first and second components obtained from the principal component analysis (PCA). Further information is described in [54].

The following analysis is focused on selecting a set of physiological features and a model able to provide similar values to the $S_{r e f}(p, x)$ at the two identified states.

\subsection{Stress Estimation}

A subset of the 27 features extracted from the physiological signals was selected according to the experiment conditions using the following criteria: (1) to show no significant differences between $\mathrm{BL}_{\mathrm{R}}$ and $\mathrm{BL}_{\mathrm{S}}$ stages' values, (because equivalent states at the end of both baseline stages were assumed) and (2) to have a correlation coefficient higher than 0.4 with the stress reference level, to ensure that the feature selected and the stress level references share at least $15 \%$ of common values (value in which the coefficient of determination is at least 0.15 ).

Furthermore, in a second step and to avoid linear dependencies and redundancies among features, Pearson's correlation between them was computed at each stage. A correlation coefficient greater than \pm 0.98 in all stages was considered to group-related features. One feature was selected per group of related features.

A linear relationship between this subset of features and the estimated stress level reference was assumed. Feature values of RS and AT stages were associated to BS and SS states, respectively. Coefficients of this linear function were computed using a linear regression model between $S_{\text {ref }}$ values at BS and SS and the values of the selected features at RS and AT. This is an alternative pathway to estimate the stress reference level using physiological signals.

Additionally, through the linear function obtained, stress level state or $S_{\text {ref }}$ was calculated for each stage of the experiment and its values were compared between participants.

\subsection{Statistical Analysis}

The participant's state at the end of the Relax and the Stress Sessions (BS and SS) was taken as the lower and higher band of moderate the stress state respectively. Variations in biochemical variables, psychometric questionnaire 
scores and stress reference levels between both sessions were analysed using the Student's t-test for paired samples as they have resulted in normally distributed data. The data were tested for normality using the Kolmogorov-Smirnov test prior to analysis.

Physiological feature values associated with each stage are the median of the feature values over the whole time stage interval (i.e. ST, MT, VD and AT) and for stages that last more than 5 min (i.e. $\mathrm{BL}_{\mathrm{R}}, \mathrm{BL}_{\mathrm{S}}$, RS and SA). The associated feature values were calculated over the last $5 \mathrm{~min}$ of the stage.

The statistical differences between the physiological feature values of two different stages were checked using the Wilcoxon signed rank test (non-parametric test), as they are considered non-normally distributed data. A Friedman's test (repeated measurements analysis of variance on ranks, non-parametric test) was performed for each feature to determine whether there was any change in the different stages of the values (a p-value $<0.05$ was considered statistically significant, meaning that there are at least two different median stage values).

The correlation between psychometric, biochemical and proposed stress reference levels with the physiological features was studied through the linear Pearson's correlation. Sessions were analysed using the Student's t test for paired samples.

\section{Results}

The study included 40 healthy young participants. The group constituted a socio-demographic homogeneous data sample, including 23 females and 17 male subjects, with mean age of $21.3 \pm 2.8$ and a body mass index of $22.1 \pm 2.7$. For the subsequent analysis, only 38 of the 40 participants were considered, since two participants did not attend the second session.

\subsection{Stress Reference Variables}

The results of the psychometric questionnaires values, the biochemical variables and calculated stress reference level are presented in Table 2. Values for the STAI-s and VASS questionnaires were statistically significantly higher in the Stress Session than in the Relax Session, thus reflecting the subjects' stress states. Results of PSS and STAI-t scales are similar in both sessions, in that they represent individuals' traits thereby indicating coherence in the experiment. Values of $\Delta$ cortisol, copeptin and prolactin were significantly different between sessions. In contrast, $\Delta \alpha$-amylase did not exhibit significant differences, although median values showed a small increase. This may be because $\alpha$-amylase concentration has a faster dynamic [55].

\subsection{Stress Reference Level}

Reference variables showing statistically significant differences between BS and SS states were utilized to produce the stress level reference scale. The contribution of each reference variable to the quantitative stress level scale was estimated based on a PCA analysis. By evaluating Eq. (1) with the estimated coefficients of the reference variables, the stress level value $\left(S_{\text {ref }}(p, x)\right)$ of participant $p$ at state $x$ was calculated as follows:

$$
S_{\text {ref }}(p, x)=0.25 \cdot \operatorname{VASS}^{\prime}(p, x)+0.25 \cdot \operatorname{STAI}^{\prime}(p, x)+0.16 \cdot \operatorname{Cortizol}^{\prime}(p, x)+0.16 \cdot \operatorname{Prolactine}^{\prime}(p, x)+0.16 \cdot \operatorname{Copeptine}^{\prime}(p, x)
$$


Table 2 Inter-subject median and median absolute deviation (MAD) of stress markers.

\begin{tabular}{|c|c|c|c|c|c|c|c|c|}
\hline \multirow[b]{3}{*}{ Stress markers } & \multicolumn{2}{|c|}{ Relax Session } & \multicolumn{6}{|c|}{ Stress Session } \\
\hline & \multirow{2}{*}{$\begin{array}{c}\text { Baseline } \\
\mathrm{BL}_{\mathrm{R}}\end{array}$} & \multirow{2}{*}{$\begin{array}{c}\text { Relax } \\
\text { RS (BS) }\end{array}$} & \multirow{2}{*}{$\begin{array}{c}\text { Baseline } \\
\mathrm{BL}_{\mathrm{s}}\end{array}$} & \multicolumn{5}{|c|}{ Trier Social Stress Test modified } \\
\hline & & & & ST & MT & SA & VD & AT (SS) \\
\hline PSS $\quad[0-40]$ & - & $21.00(2.26)$ & - & - & - & - & - & $20.00(2.57)$ \\
\hline STAI-s [0-80] & - & $12.00(7.42)$ & - & - & - & - & - & $28.0(11.72) * *$ \\
\hline \multicolumn{9}{|l|}{ Biochemical variables } \\
\hline$\Delta \alpha$-amylase $(U / m L)$ & - & $30.60(78.0)$ & - & - & - & - & - & $50.06(97.4)$ \\
\hline$\Delta$ cortisol $(\mathrm{nmol} / \mathrm{L})$ & - & $-0.08(0.09)$ & - & - & - & - & - & $0.01(0.15)$ \\
\hline Copeptine $(\mathrm{pmol} / \mathrm{L})$ & - & $6.00(3.17)$ & - & - & - & - & - & $7.60(3.74)$ \\
\hline Prolactin $(\mathrm{ng} / \mathrm{mL})$ & - & $6.34(1.65)$ & - & - & - & - & - & $8.78(4.84)$ \\
\hline
\end{tabular}

Physiology features

\begin{tabular}{|c|c|c|c|c|c|c|c|c|}
\hline \multicolumn{9}{|l|}{ Temperature } \\
\hline$\Delta \mathrm{T}_{\mathrm{fi}}\left({ }^{\circ} \mathrm{C}\right)$ & $0.15(0.14)$ & $-0.03(0.05)$ & $0.20(0.12)$ & $-0.97(0.41)$ & $-0.86(0.32)$ & $0.06(0.31)$ & $-0.35(0.22)$ & $-0.36(0.24)$ \\
\hline $\mathrm{T}_{\text {fi_Ptotal }}\left(10^{-3}{ }^{\circ} \mathrm{C}^{2} . \mathrm{s}\right)$ & $1.23(0.08)$ & $1.25(0.10)$ & $1.23(0.11)$ & $1.16(0.09)$ & $1.05(0.10)$ & $0.93(0.15)$ & $0.94(0.19)$ & $0.78(0.19)$ \\
\hline$\Delta \mathrm{T}_{\mathrm{fa}}\left({ }^{\circ} \mathrm{C}\right)$ & $0.01(0.02)^{\wedge}$ & $0.01(0.01)$ & $0.05(0.03)$ & $0.02(0.06)$ & $0.08(0.05)$ & $0.01(0.01)$ & $0.13(0.06)$ & $0.07(0.04)$ \\
\hline $\mathrm{T}_{\text {fa_Ptotal }}\left(10^{-3}{ }^{\circ} \mathrm{C}^{2} . \mathrm{s}\right)$ & $1.19(0.06)$ & $1.20(0.07)$ & $1.16(0.05)$ & $1.18(0.04)$ & $1.19(0.04)$ & $1.18(0.04)$ & $1.19(0.04)$ & $1.24(0.04)$ \\
\hline$T_{\text {Ratio }}$ & $1.00(0.05)$ & $1.02(0.03)$ & $1.04(0.05)$ & $1.00(0.03)$ & $0.94(0.05)$ & $0.89(0.07)$ & $0.89(0.10)$ & $0.80(0.07)$ \\
\hline \multicolumn{9}{|l|}{ Heart Rate } \\
\hline$H R_{\text {mean }}($ beat/min $)$ & $72.39(6.1)$ & $70.66(6.9)$ & $70.25(7.0)$ & $81.03(8.9)$ & $82.87(8.6)$ & $69.00(7.68)$ & $71.05(9.0)$ & $86.86(11.2)$ \\
\hline$R R_{S D N N}(\mathrm{~s})$ & $0.06(0.02)$ & $0.07(0.02)$ & $0.07(0.02)$ & $0.08(0.02)$ & $0.10(0.03)$ & $0.08(0.02)$ & $0.08(0.03)$ & $0.10(0.03)$ \\
\hline$R R_{r M S S D}(\mathrm{~s})$ & $0.83(0.08)$ & $0.86(0.09)$ & $0.86(0.09)$ & $0.75(0.08)$ & $0.75(0.08)$ & $0.88(0.10)$ & $0.85(0.10)$ & $0.71(0.08)$ \\
\hline$H R_{P V L F}\left(s^{-2}\right)$ & $1.85(0.33)$ & $1.77(0.32)$ & $1.78(0.36)$ & $2.39(0.48)$ & $2.43(0.44)$ & $1.72(0.34)$ & $1.90(0.44)$ & $2.65(0.64)$ \\
\hline$H R_{P L F}\left(10^{-3} \mathrm{~s}^{-2}\right)$ & $1.73(0.88)^{\wedge}$ & $1.28(0.71)$ & $1.17(0.62)$ & $1.39(0.38)$ & $2.15(0.71)$ & $1.24(0.54)$ & $1.12(0.45)$ & $2.41(0.85)$ \\
\hline$H R_{P H F}\left(10^{-3} \mathrm{~s}^{-2}\right)$ & $1.59(0.77)$ & $1.48(0.67)$ & $1.45(0.67)$ & $1.64(0.78)$ & $2.33(0.96)$ & $1.53(0.81)$ & $1.70(0.66)$ & $2.65(1.07)$ \\
\hline$H R_{L F / H F}\left(10^{2}\right)$ & $1.24(0.77)^{\wedge}$ & $0.93(0.49)$ & $0.64(0.32)$ & $0.94(0.28)$ & $1.03(0.30)$ & $0.93(0.26)$ & $0.61(0.18)$ & $0.88(0.23)$ \\
\hline \multicolumn{9}{|l|}{ Pulse Rate } \\
\hline$P R_{\text {mean }}($ beat $/$ min $)$ & $72.34(6.7)$ & $71.01(7.5)$ & $70.80(7.0)$ & $81.64(8.9)$ & $81.46(8.2)$ & $68.05(6.9)$ & $70.76(8.9)$ & $85.80(9.6)$ \\
\hline$P P_{S D N N}(s)$ & $0.07(0.02)$ & $0.09(0.03)$ & $0.07(0.02)$ & $0.10(0.02)$ & $0.13(0.03)$ & $0.10(0.03)$ & $0.10(0.03)$ & $0.12(0.02)$ \\
\hline$P P_{r M S S D}(\mathrm{~s})$ & $0.85(0.10)$ & $0.88(0.09)$ & $0.87(0.08)$ & $0.75(0.09)$ & $0.77(0.06)$ & $0.90(0.09)$ & $0.87(0.09)$ & $0.72(0.07)$ \\
\hline \multicolumn{9}{|l|}{ Pulse Wave Rising Time } \\
\hline$P R T_{\text {mean }}(\mathrm{ms})$ & $140.4(7.5)$ & $140.5(7.8)$ & $148.7(11.3)$ & $137.7(9.3)$ & $155.3(30.7)$ & $147.0(11.0)$ & $155.6(22.7)$ & $141.7(18.0)$ \\
\hline PRTSTD (ms) & $10.9(5.9)$ & $12.7(5.3)$ & $14.9(8.9)$ & $28.8(16.0)$ & $46.4(19.6)$ & $45.9(12.0)$ & $38.3(15.6)$ & $46.9(13.0)$ \\
\hline \multicolumn{9}{|c|}{ Pulse Wave Decreasing Time } \\
\hline$P D T_{\text {mean }}(\mathrm{ms})$ & $697.0(77.4)$ & $700.6(70.4)$ & $703.5(74.4)$ & $596.5(85.7)$ & $577.0(57.0)$ & $689.9(90.0)$ & $677.7(87.0)$ & $542.4(72.9)$ \\
\hline PDTsTD (ms) & $74.1(17.8)$ & $82.9(21.9)$ & $75.1(18.1)$ & $91.7(18.8)$ & $113.4(22.7)$ & $127.9(23.8)$ & $77.7(20.4)$ & $105.7(19.1)$ \\
\hline \multicolumn{9}{|c|}{ Pulse Width until Reflected wave } \\
\hline$P W r$ mean (ms) & $306.9(12.2)^{\wedge}$ & $305.9(16.8)$ & $315.7(13.3)$ & $317.1(11.7)$ & $327.4(25.3)$ & $305.1(18.3)$ & $316.5(24.0)$ & $311.1(24.1)$ \\
\hline$P W r_{S T D}(m s)$ & $18.6(7.3)$ & $17.6(9.5)$ & $20.7(7.9)$ & $35.9(14.2)$ & $53.4(17.4)$ & $59.5(16.5)$ & $42.5(13.6)$ & $52.7(9.7)$ \\
\hline \multicolumn{9}{|l|}{ Pulse Transit Time } \\
\hline$P T T_{\text {mean }}(m s)$ & 201.4(13.6) & 205.1(15.6) & $203.8(14.0)$ & $188.2(15.9)$ & $175.1(13.8)$ & $189.4(11.9)$ & $181.3(18.0)$ & $162.6(18.4)$ \\
\hline$P T T_{S T D}(m s)$ & $10.3(4.0)$ & $12.0(5.2)$ & $10.2(3.0)$ & $16.5(7.2)$ & $22.0(10.3)$ & $37.6(8.2)$ & $16.9(7.3)$ & 24.4(7.9) \\
\hline
\end{tabular}




\subsection{Stress Estimation from Physiological Features}

The most relevant features from the skin temperature, ECG and PPG signals that show variations due to the triggered stress response, according to the experimental conditions, were selected as stress markers from the whole set. The median and median absolute deviation (MAD) values of the features at the different stages of the experiment are shown in Table 2, which characterize the individuals' states at each stage of the experiment. It can be seen how most of the features change from one task to another and they have significant changes at MT and AT with respect to the other stages. Those features whose median at each stage is not significantly different from its median at RS are highlighted in Table 2. As a result of the Friedman test, each of the features has at least one difference per pair compared with their stage values $(\mathrm{p}$ value $<0.05)$ and therefore, they all changed in at least one stage of the experiment.

\subsubsection{Stress Biomarkers Selection}

At the start of each session, participants engaged a relaxation exercise to obtain a similar baseline state. Hence, at Baseline stages $\left(\mathrm{BL}_{\mathrm{R}}\right.$ and $\left.\mathrm{BL}\right)$, the feature values should not have significant differences between them. Therefore, features showing statistically different values in both baseline stages were not taken into consideration as stress markers in this experiment assuming that they may instead reflect any other difference that is not related to stress. These discarded features from the baseline stages were $\Delta \mathrm{T}_{\mathrm{fa}}, H R_{P L F}, H R_{L F / H F}$ and $P W r_{\text {mean }}$. Likewise, at stages RS and AT, considered basal stress and highest stress levels respectively, the features should vary significantly. Accordingly, those features whose medians were not significantly different between stages RS and AT (i.e. $\mathrm{T}_{\text {fi_Ptotal }}, H R_{L F / H F}$, $P R_{L F / H F}, P R T_{\text {mean }}$ and $\left.P W r_{\text {mean }}\right)$, were not taken into account as stress markers in this experiment

Table 3. Pearson's correlation between physiology parameters and stress reference variables.

\begin{tabular}{|c|c|c|c|c|c|c|c|}
\hline \multicolumn{2}{|c|}{ Features } & \multirow{2}{*}{$\frac{\text { STAI-S }}{-}$} & \multirow{2}{*}{$\frac{\text { VASS }}{-}$} & \multirow{2}{*}{$\frac{\Delta \text { Cortisol }}{-}$} & \multirow{2}{*}{$\begin{array}{c}\text { Copeptine } \\
-0.34\end{array}$} & \multirow{2}{*}{$\frac{\text { Prolactin }}{-}$} & \multirow{2}{*}{$\begin{array}{c}\mathrm{S}_{\mathrm{ref}} \\
-\end{array}$} \\
\hline Temperature & $\Delta \mathrm{T}_{\mathrm{fi}}$ & & & & & & \\
\hline & $\mathrm{T}_{\mathrm{fi} \_ \text {Ptotal }}$ & $-0.46^{*}$ & $-0.57 * *$ & -0.35 & - & -0.27 & $-0.59 * *$ \\
\hline & $\Delta \mathrm{T}_{\mathrm{fa}}$ & 0.34 & $0.40^{*}$ & $0.49 * *$ & - & 0.38 & $0.46^{* *}$ \\
\hline & $\mathrm{T}_{\text {fa_Ptota } 1}$ & 0.34 & - & 0.26 & - & - & 0.25 \\
\hline & Tratio & $-0.51 * *$ & $-0.60 * *$ & -0.39 & - & -0.26 & $-0.62 * *$ \\
\hline \multirow[t]{4}{*}{ ECG } & $H R_{\text {mean }}$ & $0.43^{*}$ & $0.47 * *$ & $0.45^{*}$ & - & $0.45^{*}$ & $0.59 * *$ \\
\hline & $R R_{S D N N}$ & - & - & - & 0.28 & - & - \\
\hline & $R R_{r M S S D}$ & -0.39 & $-0.43^{*}$ & -0.32 & - & $-0.41 *$ & $-0.51 * *$ \\
\hline & $H R_{P V L F}$ & $0.43^{*}$ & $0.47 * *$ & $0.50^{* *}$ & - & $0.48 * *$ & $0.59 * *$ \\
\hline \multirow[t]{9}{*}{ PPG } & $P R_{\text {mean }}$ & 0.34 & 0.36 & 0.35 & - & $0.42 *$ & $0.49 * *$ \\
\hline & $P P_{S D N N}$ & - & - & - & 0.28 & - & 0.32 \\
\hline & $P P_{r M S S D}$ & -0.38 & $-0.42 *$ & -0.29 & - & -0.39 & $-0.49 * *$ \\
\hline & $P D T_{\text {mean }}$ & $-0.42 *$ & $-0.46^{*}$ & -0.29 & - & -0.37 & $-0.53 * *$ \\
\hline & $P D T_{S T D}$ & - & - & - & - & - & 0.25 \\
\hline & PRTSTD & 0.29 & 0.34 & - & - & - & $0.41^{*}$ \\
\hline & PTTmean & $-0.47 * *$ & $-0.51 * *$ & $-0.41^{*}$ & - & -0.36 & $-0.63 * *$ \\
\hline & PTTSTD & 0.31 & 0.35 & 0.39 & - & - & $0.44^{*}$ \\
\hline & PWrSTD & 0.28 & 0.36 & - & - & - & $0.40^{*}$ \\
\hline
\end{tabular}

* p-value $<0.001 . * *$ p-value $<0.0001$ 
So far, for each participant, two sets of reference variable values were obtained: one at the BS (minimum stress level) and one at the SS (showing the stress level reached after the complete TSST). Using these values, linear Pearson correlations between physiological features and stress reference variables were computed. The resulting correlation coefficients are found in Table 3.

The set of features presented in Table 3 was analysed to discern features with linear dependences between them throughout this experiment, because such features will give redundant information to the stress measurement model in these specific experimental conditions. Three groups were found: (1) $\mathrm{T}_{\text {fi_Ptotal }}$ and $\mathrm{T}_{\text {ratio }}$; (2) $H R_{\text {mean }}, R R_{r M S S D}$, $H R_{P V L F}, P P_{r M S S D}$ and $P D T_{\text {mean }}$; and (3) $P P_{S D N N}$ and $P D T_{S T D}$. From each group, the feature that had the highest correlation value with stress reference level was selected. These are $\mathrm{T}_{\text {ratio, }} H R_{\text {mean }}$ and $P P_{S D N N}$.

From previously explained considerations and only including features that correlate more than 0.4 with stress reference $S_{\text {ref }}(\mathrm{r}>0.4)$, the features selected are as follow: $\mathrm{T}_{\text {ratio, }} H R_{\text {mean }}, P R T_{S T D}, P T T_{\text {mean }}, P T T_{S T D}$ and $P W r_{S T D}$.

\subsubsection{Stress Estimation}

A stress estimation function $\left(S_{e s t}\right)$ was implemented assuming a linear relation between selected features and stress level, see Eq. (3). A linear regression between $S_{\text {ref }}$ values at BS and SS and the values of the selected feature at RS and AT were computed and the coefficients extracted.

$$
S_{\text {est }}=-40.9 \cdot T_{\text {ratio }}+0.25 \cdot H R_{\text {mean }}+171.1 \cdot P R T_{\text {STD }}-145.4 \cdot P T T_{\text {mean }}-79.83 \cdot P T T_{\text {STD }}-94.1 \cdot P W r_{\text {STD }}+80.51
$$

Results of the stress level reference from reference variables against the estimated stress are presented in Fig 3, in which two well-defined groups for basal and stress states can be observed. The RMS error was 9.57 ( $\left.\mathrm{R}^{2} 0.67\right)$.

a)

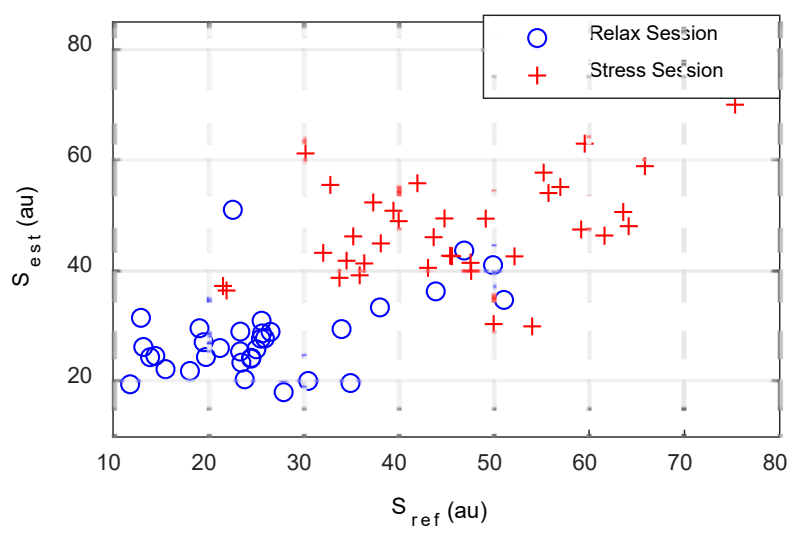

b)

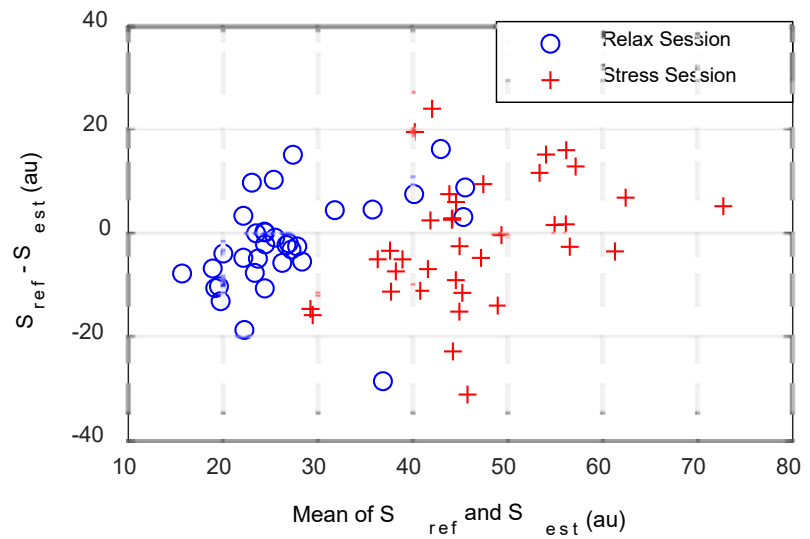

Fig. 3 Results of stress estimations. a Stress level estimated from reference variables against the estimation performed through selected features. b Black-Altman plot of the same data as in a.

The stress estimation results show an increase of $64.13 \%$ from basal to stress state generated, while the same results through the stress level reference values are $66.94 \%$. So, differences between BS and SS are in the range of $18 \%$ which, as expected, represents a moderate but significant stress effect. At basal state, the values have less 
dispersion than those of the stress state because a stressor affects individuals differently, while basal states tend to be similar.

Through the linear equation obtained Eq. (3), the stress level state was calculated for each stage of the experiment and its values are shown in Fig. 4. The stress level reached in the ST and SA task have similar median values although physiological stress markers do not have similar median values, indicating that similar stress level values could clearly be assigned to different states. The paired t test between estimated stress values for each stage was also calculated. $S_{\text {est }}$ at each of the stages related to the TSST are statistically different from the $S_{\text {est }}$ measured at relaxation stages $\left(\mathrm{BL}_{\mathrm{R}}, \mathrm{RS}\right.$ and $\left.\mathrm{BL}_{\mathrm{S}}\right)$. Table 4 shows the $\mathrm{p}$ values of the paired t test. Stages with a relaxation exercise show values of approximately $29.06 \mathrm{au}$, while those in the Stress Session rise to values ranging from 34.58 to 47.55 au.

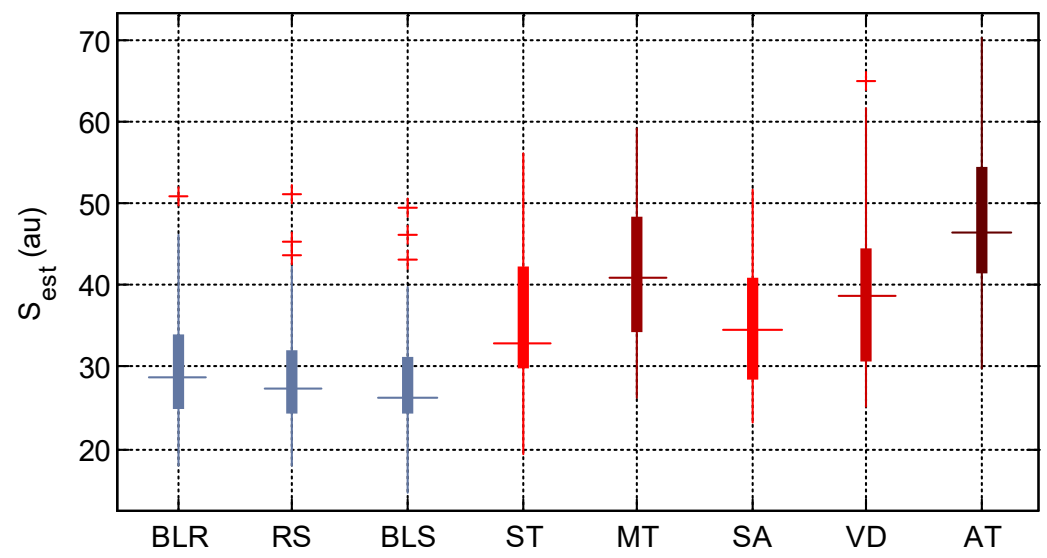

Fig. 4 Stress measurement of a participant throughout both sessions.

Table 4. $\mathrm{p}$ values of the paired t-test among measured stages' stress.

\begin{tabular}{|c|c|c|c|c|c|c|c|}
\hline \multirow[b]{2}{*}{ Stages } & \multicolumn{2}{|c|}{ Relaxed States } & \multicolumn{5}{|c|}{ Stressed States } \\
\hline & RS & BLs $_{s}$ & ST & MT & $\mathbf{S A}$ & VD & AT \\
\hline $\mathrm{BL}_{\mathrm{R}}$ & 0.125 & 0.114 & 0.006 & $2.19 \cdot 10^{-5}$ & 0.012 & $8.20 \cdot 10^{-5}$ & $5.04 \cdot 10^{-9}$ \\
\hline $\mathrm{RS}$ & & 0.538 & $3.17 \cdot 10^{-5}$ & $1.51 \cdot 10^{-7}$ & $1.82 \cdot 10^{-4}$ & $1.98 \cdot 10^{-7}$ & $4.17 \cdot 10^{-12}$ \\
\hline $\mathrm{BL}_{\mathrm{S}}$ & & & $6.14 \cdot 10^{-10}$ & $2.17 \cdot 10^{-14}$ & $1.03 \cdot 10^{-7}$ & $5.86 \cdot 10^{-13}$ & $1.87 \cdot 10^{-17}$ \\
\hline $\mathrm{ST}$ & & & & $4.54 \cdot 10^{-10}$ & 0.844 & $1.57 \cdot 10^{-4}$ & $3.50 \cdot 10^{-14}$ \\
\hline MT & & & & & $2.22 \cdot 10^{-7}$ & 0.030 & $1.25 \cdot 10^{-8}$ \\
\hline SA & & & & & & $2.57 \cdot 10^{-7}$ & $5.76 \cdot 10^{-17}$ \\
\hline VD & & & & & & & $1.67 \cdot 10^{-14}$ \\
\hline
\end{tabular}

As physiological signals were continuously recorded, all selected features were computed over a 1 min window for the whole session and then a continuous stress estimation can be performed every minute using the Eq. (3). The $S_{\text {est }}$ values from two different participants throughout both sessions are seen in Fig. 5. It shows the evolution of participants along the sessions. 

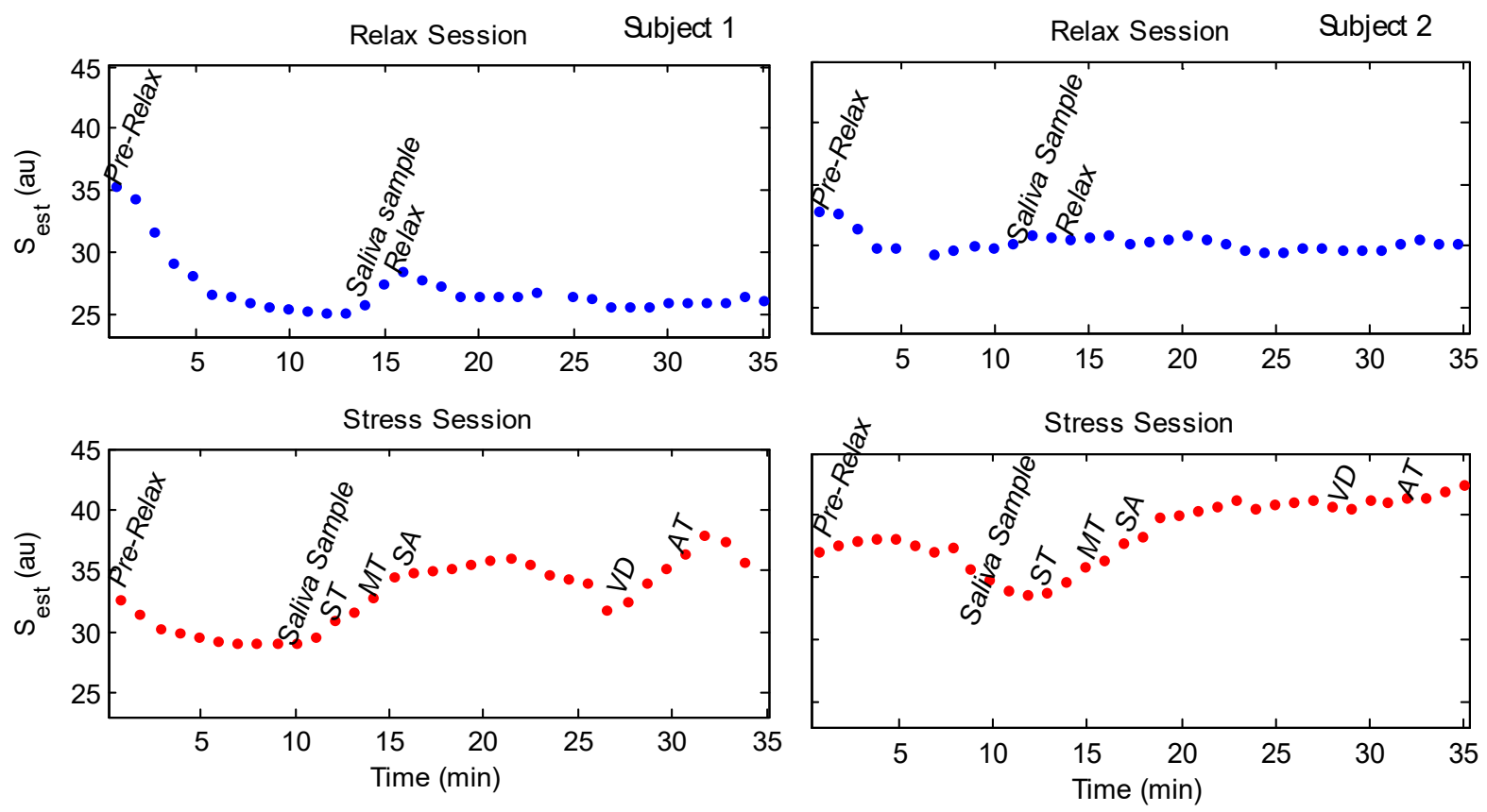

Fig. 5 Stress measurement from two subjects throughout both sessions. On the left for subject 1 and on the right for subject 2.

Figure 6 presents the evolution of other participants along the Stress Session. Different stress responses were selected among the participants to show the diversity of stress responses generated throughout the session. It can be observed that some participants had a higher stress level than others at the beginning of the session. In addition, some of them greatly reduced their values in $\mathrm{BL}_{\mathrm{S}}$, while others remained quite similar.

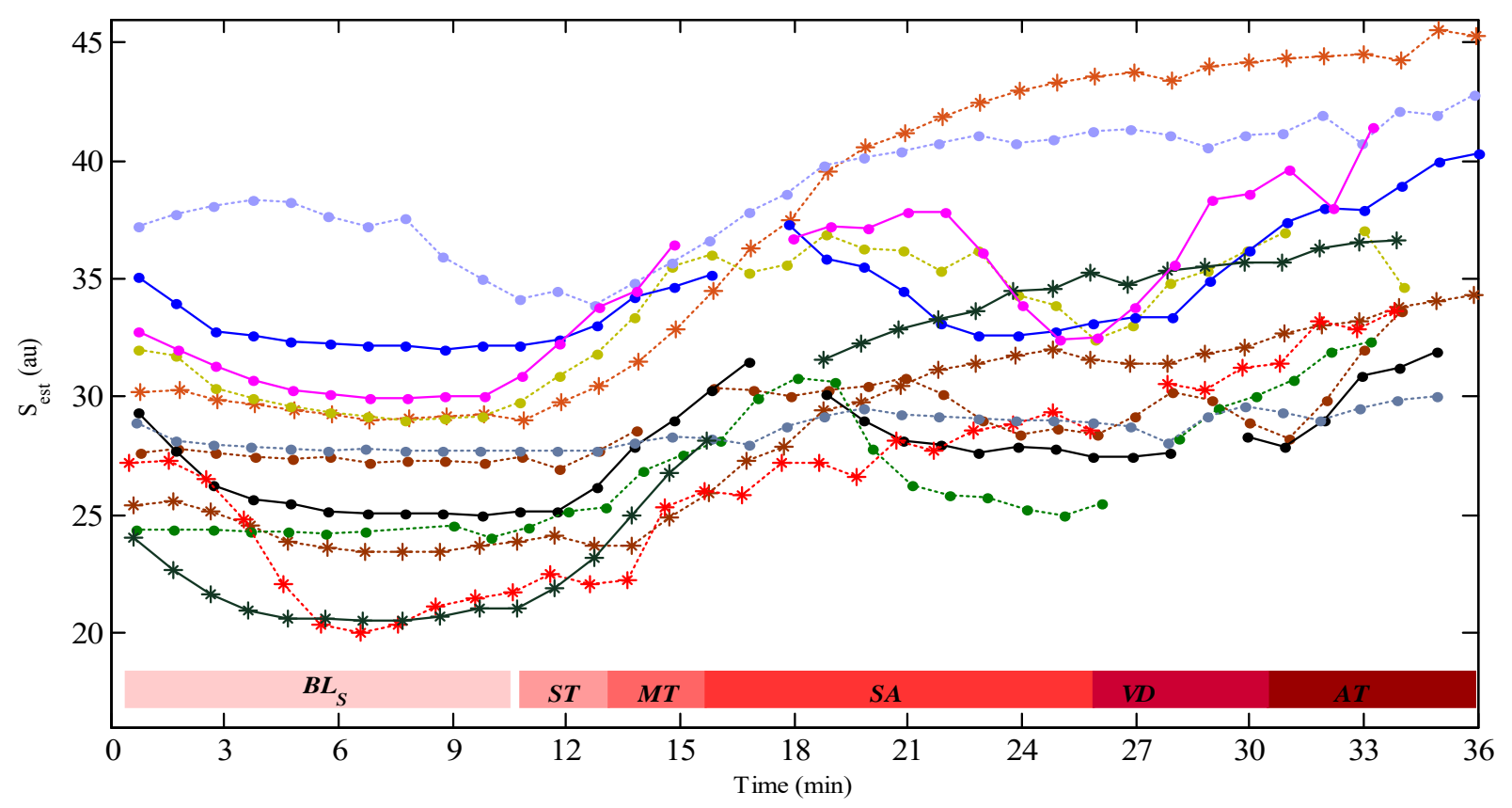

Fig. 6 Stress measurements of different subjects throughout the Stress Session. 


\section{Discussion}

The stress concept establishes that stress arises when a specific event threatens the homeostatic stability of an individual. The ultimate goal of this work is to find an approximate but quantitative measure of that homeostatic imbalance. We faced a complex human system in which the stress response elicits multiple physiological and cognitive reactions [35] in the individual. This is why none of the established stress markers alone allow for reliable quantitative assessment of stress response intensity. In this regard, we propose to use a set of biomarkers that comprehensively assess the level of stress response and then employ them in a multivariable method of stress measurement. In this way, we could have a broad measurement of the stress response and consider extensive aspects triggered by the stressor (see Table 1).

Applying the proposed multimodal approach for stress measurement, a multivariable stress reference level is proposed by merging the above-mentioned stress reference variables (see Section 3.1) into a single scale. As a first approximation, a linear relation between stress level reference and each variable was assumed. The scale of stress level reference shows higher correlations with the physiologic features than with either the psychometric variables or the biochemical variables alone. This suggests that this multivariable approach might improve the most commonly used methods. The same approach is used to estimate the acute stress response through physiological biomarkers. Moreover, in view of a possible stress monitoring method, the biomarkers selected were limited to those that can be continuously monitored and therefore they were obtained from physiological signals that are not only continuously, but also unobtrusively, measured.

The stress estimation method presented in this paper gives continuous values of acute physiological stress every 1 min. Results prove its ability not only to measure changes from a basal state (27.86 au.) to a stress state (47.55 au), but also to measure the stress level at different stress states produced throughout the sessions. This makes it possible to statistically discriminate between five stress levels induced in this experiment $\left(\mathrm{BL}_{\mathrm{R}}-\mathrm{RS}-\mathrm{BL} \mathrm{L}_{\mathrm{S}}\right.$; ST-SA; MT; VD; and AT). Moreover, although a tendency to increase the stress level in the ST and MT stages is found, in the SA and VD stages, different reactions are observed since these stages may be related to social phobia or assessment anxiety rather than stress. In the last stage, the AT also triggers different levels of stress, according to the participant's capabilities to face this task.

The presented approach can establish the groundwork for applications that aim to monitor stress and improve research in the field of stress, because it might constitute a starting point for future inter-individual and intraindividual valid comparisons of facing different stressors.

Until now, the stress response level has not been quantitatively assessed as it was above-presented and only stress detections and classifications have been made [12, 18-20]. To the best of our knowledge, there is no objective, reliable, repeatable and easily applicable method either to compare the values of the stress response level of an individual at different stressful moments or to compare the stress state of two different individuals for a fast and reliable follow-up. Indeed, current methods cannot define thresholds and have not provided quantitative and reliable estimations of the global stressing effects of an alleged stressor on a population sample. 
No clear correlation pattern between measured physiological parameters and perceived levels of stress has yet been found [3, 12]. Moreover, no agreements exist among professionals or within the research community either on a standard ground truth of stress level assessment or in a standard stress measurement method. This is probably a consequence of the variety of stimulus that induce stress and the diversity of human reactions to each stressor [20]. Additionally, it might be also a result of the fact that the extant literature aims to assess one or very few reactions that arise from the stress response, rather than comprehensively describe the physiological stress response itself as we propose.

We used a reliable protocol and a well-documented stressor such as the TSST [37]. The purpose was to induce moderate, but significant, stress with a validated and widely documented tool for the generation of stress [16]. Results of both physiological features and stress reference values provide evidence of the two different stress levels of induced states, BS and SS, in agreement with previous studies [16, 37].

Features extracted from finger and face skin temperatures, as well as electrocardiogram and pulse wave signals, were selected to comprehensively identify the physiological stress response. Changes related to stress that these selected signals have are seen in table I (Section 2.4). Most of the features exhibit statistically different values between the BS and the SS and a significant linear correlation with the reference variables, as has been reported in others studies [16]. HR features are highly correlated with the stress reference level, as well as PR features, which are quite similar to results presented in [56]. A preliminary analysis of heart rate variability (HRV) as a stress marker has also already been reported in [43]. Nevertheless, respiratory information should be taken into account to increase the reliability of HRV as a marker of stress [57] as frequency features of HRV are influenced by respiration [58-60]. This analysis would be an important addition to futures studies.

Meanwhile, other features, such as $T_{\text {ratio, }} P R T$ and PTT, are first used as stress biomarkers in this study. In the BL and RS stages, both finger and face temperatures, show low variations following the normal body thermoregulation $\left(T_{\text {ratio }} \approx 1\right)$, but in stages in which a stressor is applied, for instance at ST, MT, VD and AT, face temperature increases while finger temperature decreases. Similar results are reported in [25]. PTT mean has the highest Pearson correlation value with the stress reference level and it is also highly sensitive to the different states induced throughout the experiment. The lower $P T T_{\text {mean }}$ values occurs for stressful tasks, indicating an increase in both blood vessel resistance and blood pressure, while the highest $P T T_{\text {mean }}$ values occur for relaxing tasks.

The experiment described in this paper takes a particular sample (healthy young people) that was subjected to a particular stress stimulus, TSST. However, other physiological responses should be investigated using other groups of individuals facing another stressor. Moreover, in a real-life application, environmental conditions must be included, as well as certain characteristics such as age, gender, IMC, physical activity and diseases. In real-life conditions, more complex algorithms than the presented estimation of stress should be used, in which the previously mentioned conditions should be included.

Furthermore, only six features from the whole set have been retained, applying above explained selection criteria. However, it is important to note that this selection was tailored to these restricted experimental conditions, in which healthy young participants were sitting and only under the influence of the experimental tasks. Those restrictions 
were meant to eliminate from the equation features that, in this case, do not provide additional information or have not shown significant variations. When applying this method to a population with other characteristics and subjected to another stressor, however, these simplifications will not be valid and others will probably be applicable. Then, this method will provide, for example, a quantitative assessment of how far an individual is from the average of this population. Therefore, changes in features other than those selected in this experiment could be triggered by either other stressors or in different conditions than the ones used in this experiment, as well as acting in a different population sample or when a more intensive stress response arises.

As previously mentioned, the correlation analysis between physiological features reveals that some of them were changing in equal proportions during this experiment. Even if they met the selection criteria, only one feature per related group was used for the estimation performed, as their information was redundant. However, all others could be newly included in future studies under different conditions, such as real situations or long-term stress monitoring.

There are also some limitations to note regarding this study. Only linear relations between the stress response and the stress reference variables were analysed in this preliminary approach. Even though the results obtained strongly support this approach, other approaches, including non-linear relations could probably yield better results. Likewise, it was assumed that the stress function is linear in the six features selected, but slightly different features and relations could be analysed in future research to improve on these results. A limitation of the protocol is that the relaxation period is restricted to only $10 \mathrm{~min}$ and participants may need a longer time to physiologically accommodate to the experimental environment. The study design has two limitations concerning biochemical timing collection: (1) cortisol and alpha-amylase are measured in the morning hours when intra-individual variability is at its highest; and (2) alpha-amylase is known to peak at about 10-15 min after stressor onset, whereas it was assessed 25 min after the stressor onset.

Despite these limitations, this laboratory based experiment can provide a solid foundation for daily life stress monitoring, by first identifying the score function adapted to a particular individual and then by applying this function to monitor daily life stress.

\section{Conclusions}

A multivariable approach based on stress markers was proposed to estimate stress levels in order to obtain a broad measurement of the stress response, thus considering the multiple reactions triggered by the stressor. This approach may establish the groundwork for future applications that aim to monitor stress. It also benefits research in the field of stress by enabling intra-individual and inter-individual evaluations to be made using a common index of stress level.

The performed experiment proves that the level of stress response caused by a psychological stressor can be determined from a set of unobtrusively and continuously measured physiological parameters, as an alternative to the currently used stress assessment methods. 
An innovative pioneering approach was proposed to estimate stress using a multivariable biomarker, constituted by features extracted from physiological signals. Results from this approach demonstrated its ability to continuously measure stress because different stages that occur sequentially in a short period of time can be easily distinguished.

A reliable and continuous stress measurement will be a useful tool for mental health care and well-being. It will also enable professionals to possess easy and precise communication methods in order to identify more suitable and personalized solutions for the treatment of pathological cases.

The results of the experiment presented in this article properly motivate further experiments with different stressors and populations in order to validate its extrapolation capabilities and to adapt its use to other conditions. Therefore, a new and more sensitive stress measurement method could emerge based on the presented results, which could be utilized effectively for unobtrusive stress monitoring.

Acknowledgments: This research was supported by MINECO (FIS-PI12/00514 and TIN2014-53567-R) and by the Centro de Investigación Biomédica en Red sobre Bioingeniería, Biomateriales y Nanomedicina (CIBER-BBN) at the Instituto de Salud Carlos III de España. This project has received funding from the European Union's Framework Programme for Research and Innovation Horizon 2020 (2014-2020) under the Marie Sklodowska-Curie Grant Agreement No. 745755.

ETHICAL APPROVAL: All procedures performed in this study involving human participants were in accordance with the ethical standards of the institutional and/or national research committee and with the 1964 Helsinki declaration and its later amendments or comparable ethical standards.

\section{References}

1. Cohen, S., Janicki-deverts, D., \& Miller, G. E. (2007). Psychological Stress and Disease. JAMA - Journal of the American Medical Association, 298(14), 1685-1687. https://doi:10.1001/jama.298.14.1685

2. American Psychological Association. (2013). By the Numbers: A Psychologically Healthy Workplace Fact Sheet. Retrieved from http://www.apaexcellence.org/resources/goodcompany/newsletter/article/487

3. Epel, E. S., Crosswell, A. D., Mayer, S. E., Prather, A. A., Slavich, G. M., Puterman, E., \& Mendes, W. B. (2018). More than a feeling: A unified view of stress measurement for population science. Frontiers in Neuroendocrinology. https://doi:10.1016/J.YFRNE.2018.03.001

4. Lagraauw, H. M., Kuiper, J., \& Bot, I. (2015). Acute and chronic psychological stress as risk factors for cardiovascular disease: Insights gained from epidemiological, clinical and experimental studies. Brain, Behavior and Immunity, 50, 18-30. https://doi:10.1016/J.BBI.2015.08.007

5. Mariotti, A. (2015). The effects of chronic stress on health: new insights into the molecular mechanisms of brain-body communication. Future Science OA, 1(3), fso.15.21. https://doi:10.4155/fso.15.21

6. Tamashiro, K. L., Sakai, R. R., Shively, C. A., Karatsoreos, I. N., \& Reagan, L. P. (2011). Chronic stress, metabolism and metabolic syndrome. Stress, 14(5), 468-474. https://doi:10.3109/10253890.2011.606341

7. World Health Organization. (2015). WHO | Mental Health Atlas 2014. WHO, 69. https://doi:978 9241565011

8. Aguilo, J., Ferrer Salvans, P., Garcia Rozo, A., Armario, A., Corbi, A., Cambra, F. J., ... Garzon Rey, J. M. (2015). Project ES3: attempting to quantify and measure the level of stress. Revista de neurologia, 61(9), 405-415. https://www.ncbi.nlm.nih.gov/pubmed/26503316

9. Selye, H. (1950). Stress and the General adaptation syndrome. British Medical Journal, 1383-1392.

10. Lazarus, R. S. (1993). From psychological stress to the emotions: a history of changing outlooks. Annual review of psychology, 44, 121. https://doi:10.1146/annurev.ps.44.020193.000245

11. Hellhammer, D. H., Stone, A. A., Hellhammer, J., \& Broderick, J. (2010). Measuring Stress. In Encyclopedia of Behavioral Neuroscience (Vol. 2, pp. 186-191). Elsevier Ltd.

12. Goyal, A., Singh, S., Vir, D., \& Pershad, D. (2016). Automation of Stress Recognition Using Subjective or Objective Measures. Psychological Studies, 61(4), 348-364. https://doi:10.1007/s12646-016-0379-1

13. Khoulji, S., García, E., Aguiló, S., Arza, A., Garzón-Rey, J. M., \& Aguilóa, J. (2017). Psychological and Physiological Profiles in Oncology Caregivers: A Multivariable Cross-Sectional Study. Transactions on Machine Learning and Artificial Intelligence, 5(4). https://doi:10.14738/tmlai.54.3291

14. Urwyler, S. A., Schuetz, P., Sailer, C., \& Christ-Crain, M. (2015). Copeptin as a stress marker prior and after a written examination the CoEXAM study. Stress (Amsterdam, Netherlands), 1-4. https://doi:10.3109/10253890.2014.993966

15. Nater, U. M., Ditzen, B., Strahler, J., \& Ehlert, U. (2013). Effects of orthostasis on endocrine responses to psychosocial stress. 
International Journal of Psychophysiology, 90(3), 341-6. https://doi:10.1016/j.ijpsycho.2013.10.010

16. Allen, A. P., Kennedy, P. J., Cryan, J. F., Dinan, T. G., \& Clarke, G. (2014). Biological and psychological markers of stress in humans: focus on the Trier Social Stress Test. Neuroscience and biobehavioral reviews, 38, 94-124. https://doi:10.1016/j.neubiorev.2013.11.005

17. Armario, A., Marti, O., Molina, T., de Pablo, J., \& Valdes, M. (1996). Acute stress markers in humans: response of plasma glucose, cortisol and prolactin to two examinations differing in the anxiety they provoke. Psychoneuroendocrinology, 21(1), 17-24. https://www.ncbi.nlm.nih.gov/pubmed/8778900

18. Campbell, J., \& Ehlert, U. (2012). Acute psychosocial stress: does the emotional stress response correspond with physiological responses? Psychoneuroendocrinology, 37(8), 1111-34. https://doi:10.1016/j.psyneuen.2011.12.010

19. Alberdi, A., Aztiria, A., \& Basarab, A. (2016). Towards an automatic early stress recognition system for office environments based on multimodal measurements: A review. Journal of Biomedical Informatics, 59(C), 49-75. https://doi:10.1016/j.jbi.2015.11.007

20. Oken, B. S., Chamine, I., \& Wakeland, W. (2015). A systems approach to stress, stressors and resilience in humans. Behavioural brain research, 282, 144-54. https://doi:10.1016/j.bbr.2014.12.047

21. Chen, L. lan, Zhao, Y., Ye, P. fei, Zhang, J., \& Zou, J. zhong. (2017). Detecting driving stress in physiological signals based on multimodal feature analysis and kernel classifiers. Expert Systems with Applications, 85, 279-291.

22. Han, L., Zhang, Q., Chen, X., Zhan, Q., Yang, T., \& Zhao, Z. (2017). Detecting work-related stress with a wearable device. Computers in Industry, 90, 42-49. https://doi:10.1016/j.compind.2017.05.004

23. Gjoreski, M., Luštrek, M., Gams, M., \& Gjoreski, H. (2017). Monitoring stress with a wrist device using context. Journal of Biomedical Informatics, 73, 159-170. https://doi:10.1016/J.JBI.2017.08.006

24. Mahmoud, R., Shanableh, T., Bodala, I. P., Thakor, N. V., \& Al-Nashash, H. (2017). Novel Classification System for Classifying Cognitive Workload Levels Under Vague Visual Stimulation. IEEE Sensors Journal, 17(21), 7019-7028. https://doi:10.1109/JSEN.2017.2727539

25. Vinkers, C. H., Penning, R., Hellhammer, J., Verster, J. C., Klaessens, J. H. G. M., Olivier, B., \& Kalkman, C. J. (2013). The effect of stress on core and peripheral body temperature in humans. Stress, 16(5), 520-30. https://doi:10.3109/10253890.2013.807243

26. Cvetković, B., Gjoreski, M., Šorn, J., Maslov, P., Kosiedowski, M., Bogdański, M., ... Luštrek, M. (2017). Real-time physical activity and mental stress management with a wristband and a smartphone. In Proceedings of the 2017 ACM International Joint Conference on Pervasive and Ubiquitous Computing and Proceedings of the 2017 ACM International Symposium on Wearable Computers on UbiComp '17 (pp. 225-228). New York, New York, USA: ACM Press. https://doi:10.1145/3123024.3123184

27. Picard, R. R. W., Fedor, S., \& Ayzenberg, Y. (2014). Multiple arousal theory and daily-life electrodermal activity asymmetry. Emotion Review.

28. Kukolja, D., Popović, S., Horvat, M., Kovač, B., \& Ćosić, K. (2014). Comparative analysis of emotion estimation methods based on physiological measurements for real-time applications. International Journal of Human Computer Studies, 72(10-11), 717-727. https://doi:10.1016/j.ijhcs.2014.05.006

29. Healey, J. A. J. A. J. a., \& Picard, R. W. R. W. R. W. (2005). Detecting Stress During Real-World Driving Tasks Using Physiological Sensors. IEEE Transactions on Intelligent Transportation Systems, 6(2), 156-166. https://doi:10.1109/TITS.2005.848368

30. Seoane, F., Mohino-Herranz, I., Ferreira, J., Alvarez, L., Buendia, R., Ayllón, D., ... Gil-Pita, R. (2014). Wearable Biomedical Measurement Systems for Assessment of Mental Stress of Combatants in Real Time. Sensors, 14(4), 7120-7141. https://doi:10.3390/s140407120

31. Katsis, C. D., Katertsidis, N. S., \& Fotiadis, D. I. (2011). An integrated system based on physiological signals for the assessment of affective states in patients with anxiety disorders. In Biomedical Signal Processing and Control (Vol. 6, pp. 261-268). https://doi:10.1016/j.bspc.2010.12.001

32. Broek, E. L., Sluis, F., \& Dijkstra, T. (2013). Cross-validation of bimodal health-related stress assessment. Personal and Ubiquitous Computing, 17(2), 215-227. https://doi:10.1007/s00779-011-0468-z

33. de Santos Sierra, A., Sanchez Avila, C., Guerra Casanova, J., Bailador del Pozo, G., Sierra, A. D. S., Ávila, C. S., ... Bailador, G. (2011). A Stress-Detection System Based on Physiological Signals and Fuzzy Logic. IEEE Transactions on Industrial Electronics, 58(10), 4857-4865. https://doi:10.1109/TIE.2010.2103538

34. Hellhammer, J., \& Schubert, M. (2012). The physiological response to Trier Social Stress Test relates to subjective measures of stress during but not before or after the test. Psychoneuroendocrinology, 37(1), 119-124.

35. Baumeister, R., \& Vohs, K. (2007). Encyclopedia of Social Psychology. (E. Harmon-Jones \& P. Winkielman, Eds.)Social Neuroscience. California: SAGE Publications, Inc.

36. Jönsson, P., Wallergård, M., Osterberg, K., Hansen, A. M., Johansson, G., \& Karlson, B. (2010). Cardiovascular and cortisol reactivity and habituation to a virtual reality version of the Trier Social Stress Test: a pilot study. Psychoneuroendocrinology, 35(9), 1397-403. https://doi:10.1016/j.psyneuen.2010.04.003

37. Kudielka, B. M., Hellhammer, D. H., \& Kirschbaum, C. (2007). Ten years of research with the Trier Social Stress Test. In Social Neuroscience (pp. 56-83). https://doi:10.4135/9781412956253.n539

38. Giles, G. E., Mahoney, C. R., Brunyé, T. T., Taylor, H. A., \& Kanarek, R. B. (2014). Stress effects on mood, HPA axis and autonomic response: Comparison of three psychosocial stress paradigms. PLOS ONE, 9(12), e113618. https://doi:10.1371/journal.pone.0113618

39. Rabasa, C., Gagliano, H., Pastor-Ciurana, J., Fuentes, S., Belda, X., Nadal, R., \& Armario, A. (2015). Adaptation of the hypothalamus-pituitary-adrenal axis to daily repeated stress does not follow the rules of habituation: A new perspective. Neuroscience \& Biobehavioral Reviews, 56, 35-49. https://doi:10.1016/J.NEUBIOREV.2015.06.013

40. UAB. (2014). ES3 stress measuring project| Universitat Autònoma de Barcelona. Retrieved June 17, 2014, from http://www.es3project.es/

41. Stetter, F., \& Kupper, S. (2002). Autogenic Training: A Meta-Analysis of Clinical Outcome Studies. Applied Psychophysiology and Biofeedback, 27(1), 45-98. https://doi:10.1023/A:1014576505223

42. Pujol, J., Giménez, M., Ortiz, H., Soriano-Mas, C., López-Solà, M., Farré, M., ... Martín-Santos, R. (2013). Neural response to the observable self in social anxiety disorder. Psychological medicine, 43(4), 721-31. https://doi:10.1017/S0033291712001857 
43. Arza, A., Garzón, J. M., Hemando, A., Aguiló, J., Bailon, R., Garzon, J. M., .. Bailon, R. (2015). Towards an objective measurement of emotional stress : Preliminary analysis based on heart rate variability. In Medicine and Biology Society (EMBC), 2015 37th Annual International Conference of the IEEE (pp. 3331-3334). IEEE. https://doi:10.1109/EMBC.2015.7319105

44. Sörnmo, L., \& Laguna, P. (2005). Bioelectrical Signal Processing in Cardiac and Neurological Applications. Academic Press.

45. Gil, E., María Vergara, J., \& Laguna, P. (2008). Detection of decreases in the amplitude fluctuation of pulse photoplethysmography signal as indication of obstructive sleep apnea syndrome in children. Biomedical Signal Processing and Control, 3(3), 267-277. https://doi:10.1016/j.bspc.2007.12.002

46. Elgendi, M. (2012). On the analysis of fingertip photoplethysmogram signals. Current cardiology reviews, 8(1), 14-25. https://www.ncbi.nlm.nih.gov/pubmed/22845812

47. Arza, A., Lazaro, J., Gil, E., Laguna, P., Aguilo, J., \& Bailon, R. (2013). Pulse transit time and pulse width as potential measure for estimating beat-to-beat systolic and diastolic blood pressure. In Computing in Cardiology Conference (CinC) (pp. 887-890). IEEE.

48. Allen, J. (2007). Photoplethysmography and its application in clinical physiological measurement. Physiological measurement, 28(3), R1-39. https://doi:10.1088/0967-3334/28/3/R01

49. Lázaro, J., Gil, E., Bailón, R., Mincholé, A., \& Laguna, P. (2013). Deriving respiration from photoplethysmographic pulse width. Medical \& biological engineering \& computing, 51(1-2), 233-42. https://doi:10.1007/s11517-012-0954-0

50. Cohen, S., Kamarck, T., \& Mermelstein, R. (1983). A Global Measure of Perceived Stress. Journal of health and social behavior, 24(4), 385-396.

51. Spielberger, C. D. (2010). State-Trait Anxiety Inventory. In The Corsini Encyclopedia of Psychology (pp. 1-1). Hoboken, NJ, USA: John Wiley \& Sons, Inc. https://doi:10.1002/9780470479216.corpsy0943

52. Nater, U. M., Rohleder, N., Schlotz, W., Ehlert, U., \& Kirschbaum, C. (2007). Determinants of the diurnal course of salivary alphaamylase. Psychoneuroendocrinology, 32(4), 392-401. https://doi:10.1016/j.psyneuen.2007.02.007

53. Jozami Guldberg, L. S. (2014). Estrés psicosocial agudo: Efectos sobre el cortisol y a- amilasa en saliva. Universidad Autónoma de Barcelona.

54. Garzon-Rey, J. M., Arza, A., De-la-Camara, C., Lobo, A., Armario, A., \& Aguilo, J. (2017). An approach to an acute emotional stress reference scale. Revista de Neurologia, 64(12), 529-537. https://www.ncbi.nlm.nih.gov/pubmed/28608352

55. Engert, V., Vogel, S., Efanov, S. I., Duchesne, A., Corbo, V., Ali, N., \& Pruessner, J. C. (2011). Investigation into the crosscorrelation of salivary cortisol and alpha-amylase responses to psychological stress. Psychoneuroendocrinology, 36(9), 1294-302. https://doi:10.1016/j.psyneuen.2011.02.018

56. Gil, E., Orini, M., Bailón, R., Vergara, J. M., Mainardi, L., \& Laguna, P. (2010). Photoplethysmography pulse rate variability as a surrogate measurement of heart rate variability during non-stationary conditions. Physiological measurement, 31(9), 1271-90. https://doi:10.1088/0967-3334/31/9/015

57. Hernando, A., Lázaro, J., Gil, E., Arza, A., Garzón, J. M., López-Antón, R., ... Bailon, R. (2016). Inclusion of respiratory frequency information in heart rate variability analysis for stress assessment. IEEE Journal of Biomedical and Health Informatics, 20(4), 1-1. https://doi:10.1109/JBHI.2016.2553578

58. Billman, G. E. (2013). The LF/HF ratio does not accurately measure cardiac sympatho-vagal balance. Frontiers in physiology, 4 , 26. https://doi:10.3389/fphys.2013.00026

59. Task Force of the European Society of Cardiology and the North American Society of Pacing and Electrophysiology. (1996). Heart rate variability: standards of measurement, physiological interpretation and clinical use. Task Force of the European Society of Cardiology and the North American Society of Pacing and Electrophysiology. Circulation, 93(5), 1043-1065. https://doi:10.1161/01.cir.93.5.1043

60. Hernando, D., Hernando, A., Casajús, J. A., Laguna, P., Garatachea, N., \& Bailón, R. (2018). Methodological framework for heart rate variability analysis during exercise: application to running and cycling stress testing. Medical \& Biological Engineering \& Computing, 56(5), 781-794. https://doi:10.1007/s11517-017-1724-9

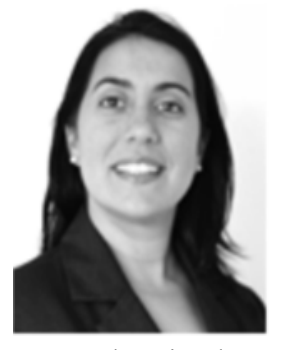

Adriana Arza Valdés is a postdoctoral researcher in the Embedded Systems Laboratory at the École Polytechnique Fédérale de Lausanne, Switzerland. She received a B.Sc. degree in Automatic Engineering from Polytechnic University of Havana, Cuba in 2005, a Master and Ph.D. degree in Microelectronic and Electronic Systems, from Autonomous University of Barcelona (UAB), Spain in 2012 and 2017, respectively. Her research interests include biological signal processing and analysis and interpretation of physiological data related to human stress measurement.

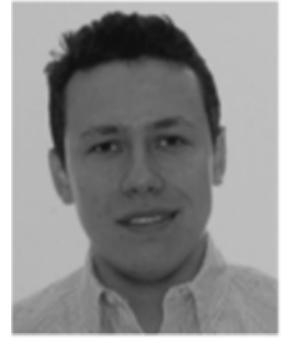

Jorge Mario Garzón Rey is a researcher in the Microelectronics and Electronic Systems Department at UAB. He received a B.Sc. in Electrical Engineering and a B.Sc. in Electronics Engineering from Universidad de los Andes (Colombia). He also received a M.Sc. in Electronics and Computer Engineering from the same University. In 2017, he received the Ph.D. degree in Telecommunication and Electronics Engineering at UAB (Spain). 


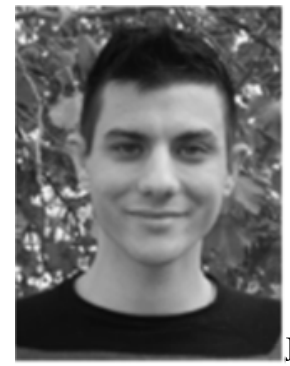

Jesús Lázaro is currently working with the Department of Biomedical Engineering of the University of Connecticut, Storrs, CT, U.S.A. and with the Aragón Institute of Engineering Research (I3A) of the University of Zaragoza (UZ), Zaragoza, Spain. He received the Master's degree in Biomedical Engineering and the Ph.D. degree in Biomedical Engineering from UZ, in 2012 and 2015, respectively. His current research interests include the field of biomedical signal processing, especially in the analysis of electrocardiographic and pulse wave signals for quantification of cardiovascular and respiratory systems.

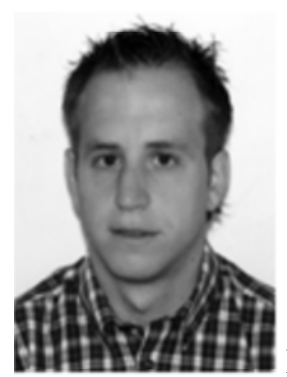

Eduardo Gil is Assistant Professor with the University of Zaragoza (UZ) and works with the I3A and with the Biomedical Research Networking Centre in Bioengineering, Biomaterials and Nanomedicine. He received the M.S. degree in telecommunication engineering and the $\mathrm{Ph} . \mathrm{D}$. degree in biomedical engineering from UZ, Spain, in 2002 and 2009, respectively. His current research interests include the field of biomedical signal processing, especially in the analysis of pulse wave signals for quantification of cardiovascular and respiratory systems.

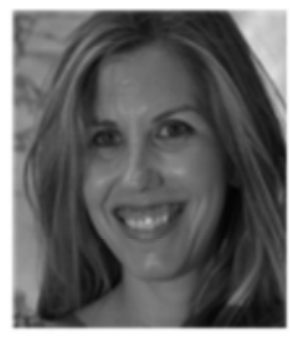

Raquel Bailón is an Associate Professor in the Department of Electronic Engineering and Communications, University of Zaragoza (UZ) and Researcher with the I3A and CIBER-BBN, Spain. She received the M.S. degree in Telecommunication Engineering and the Ph.D. degree in Biomedical Engineering from the UZ, Spain, in 2001 and 2006, respectively. Her current research interests include the biomedical signal-processing field, especially in the analysis of the dynamics and interactions of cardiovascular signals.

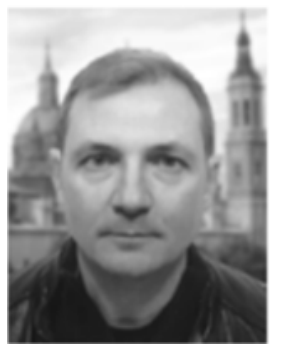

Raul Lopez-Anton is currently a Researcher at the Instituto Aragonés de Ciencias de la Salud and in the Instituto de Investigaciones Sanitarias de Aragón (Zaragoza, Spain) and an Assistant Professor in the Department of Psychology and Sociology in the University of Zaragoza, since 2014. He received his bachelor's degree in Psychology from de Universidad Nacional de Educación a Distancia and the Ph.D. degree in Psychiatry, Neurology and Neuroscience from the University of Zaragoza, Spain, in 1999 and 2011, respectively. His current research interests include neurocognitive science and the psycho-physiological measurement of stress.

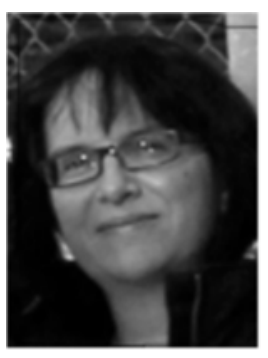

Concepción De la Cámara Izquierdo received the M.S. degree in Medicine and Surgery in 1989 and the Ph.D. degree in Medicine in 1999 from the University of Zaragoza, Spain. She is a specialist in psychiatry and currently is working as a psychiatrist in the Hospital Clínico Universitario de Zaragoza, Spain and as an Associate Professor in the Faculty of Medicine at the University of Zaragoza. She develops their research in the fields of psychogeriatric treatment, dementia, depression and stress.

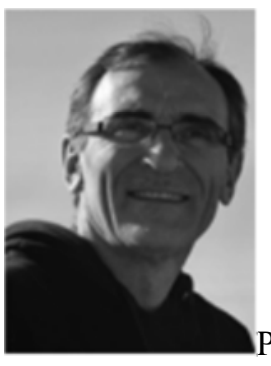

Pablo Laguna is full Professor of Signal Processing and Communications at the University of Zaragoza. His professional research interests are in signal processing applied to biomedical applications. He has coauthored more than 130 research papers on this topic, over 250 international conference papers and has advised 12 Ph.D. theses. He is a Fellow of the IEEE. 


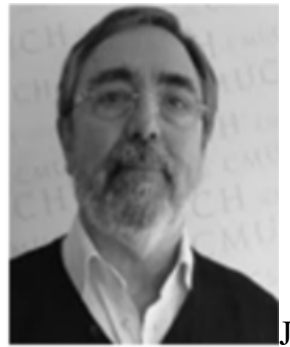
Computer Technology and Architecture at the UAB. He is leading the GBIO research group together with the
Microelectronics National Centre, CNM-CSIC. He is coordinating the bioengineering program at the CIBER-BBN. His main fields of interest are micro and nano systems and multimodal analysis for biomedical applications, mainly focusing mental disorders, well-being and personal autonomy. He has led various European projects and participated in many others, among them MEDICS: The European Centre of Competence for Biomedical Devices. $\mathrm{He}$ is currently participating in the IMI RADAR-CNS Project. 